\title{
New Discussion of the Spin Dynamics of Paramagnetic EuO
}

\author{
Z. LATACZ \\ Marian Smoluchowski Institute of Physics \\ Jagiellonian University, Reymonta 4, 30-059 Kraków, Poland \\ (Received January 2, 2004; revised version May 17, 2004; \\ in final form August 17, 2004)
}

\begin{abstract}
The simplest approximations for a continued-fraction representation of the dynamic relaxation function are used to investigate the spin dynamics of paramagnetic EuO. As the result we can see how the considered approximations predict those wavevectors for which a "spin wave" peak appears in the spectrum. Furthermore, the calculated position of this peak is compared with experiment. The correlation theory within the two-pole approximation is presented in a simple and analytic form.
\end{abstract}

PACS numbers: 75.40.Gb, 75.40.Cx, 75.50.Dd

\section{Intruduction}

The properties of EuO, the ferromagnetic material $\left(T_{\mathrm{C}}=69.2 \mathrm{~K}\right)[1-3]$ are well known as they have been investigated for a long time. However, as it usually happens, some problems are left unsolved. One of them is the problem of predicting correctly those wavevectors for which a "spin wave" peak appears in the spectrum in the paramagnetic state. From the theoretical point of view, it is well known that the dynamic properties of the system are well described by means of the continued-fraction representation of the dynamic relaxation function $\left(\boldsymbol{A}_{\boldsymbol{q}} \boldsymbol{A}_{-\boldsymbol{q}}\right)_{z}$ [4]. However, this theory is difficult except for simple systems. Therefore, in practice the following approximations for the dynamic relaxation function are used: (a) a Lorentzian approximation, (b) a two-pole approximation, (c) a cut-off two-pole approximation, (d) a three-pole approximation. The frequency and wavevector dependence of the line-shape function $F(\boldsymbol{q}, \omega)$ in the critical and paramagnetic state can be also calculated for a Heisenberg ferromagnet using the mode-coupling theory $[5,6]$. In paper $[6]$ the mode-coupling theory was compared with experimental values for $\mathrm{EuO}$ (for small values of $\boldsymbol{q}$ at $q=0.15 \AA^{-1}$ and $\left.q=0.3 \AA^{-1}\right)$. In this case good agreement was obtained. In paper [6] the calculations for EuO showed that this theory correctly describes $F(\boldsymbol{q}, \omega)$ at $T=1.68 T_{\mathrm{C}}$ and $q=q_{\max }=1.0 \AA^{-1}$ ( $q_{\max }$ is the zone-boundary wavevector in direction), but 
does not predict the paramagnetic peak at $T=T_{\mathrm{C}}$ and $q=q_{\max }=1.0 \AA^{-1}$, also it does not predict the inelastic shoulder in the line shape at the zone boundary. Instead, both theories, the correlation theory within the two-pole approximation and three-pole approximation predict the existence of the paramagnetic peak. The mode-coupling theory is very difficult from numerical point of view. In this theory the following approximations are applied $[6,7]$ :

- the dynamical mode-mode approximation for the dynamical relaxation function of four-spin operators (as in the case of the correlation theory within a two-pole approximation);

- the spherical method (random phase approximation (RPA) method) for the static properties of the system (as in the case of the three-pole approximation).

Therefore, the mode-coupling theory will not be used in the present calculations. In the present paper the results of the above-mentioned approximations, namely (b), (c), (d), will be compared for two quantities:

(1) $q^{*}$ which is the wavevector above which a "spin wave" peak appears in $F(\boldsymbol{q}, \omega)$ as a function of $\omega$ for a fixed $\boldsymbol{q}$ and $T$ (the results are shown for $\boldsymbol{q}$ in the $[1,1,1]$ direction, and are presented as a fraction of $\left.q_{\max }\right)$,

(2) the energy of the "spin wave" peak in the neutron scattering spectrum of $\mathrm{EuO}$ at a given $T$ for $q=q^{*}$.

The experimental results are from Refs. [8] and [9]. In the present paper the correlation theory within a two-pole approximation is presented in a simple and analytic form.

\section{2. $\mathrm{EuO}$}

The Heisenberg Hamiltonian for EuO can be written as $[1,10]$ :

$$
H=\sum_{i, j} J_{i j} S_{i} S_{j},
$$

where $\boldsymbol{S}_{\boldsymbol{i}}$ is a spin operator $(S=7 / 2)$ on a site of an fcc lattice. Each distinct pair in Eq. (1) is counted once and $J_{\boldsymbol{i} \boldsymbol{j}}$ are the exchange interactions. The experimental values of the nearest (NN) and next nearest neighbour (NNN) interactions (denoted by $J_{1}$ and $J_{2}$, respectively) are given in paper [10]. In the case of $\mathrm{EuO}$ the Fourier transform of $J_{\boldsymbol{i} \boldsymbol{j}}, J_{\boldsymbol{q}}$ is given by the following formula:

$$
\begin{aligned}
J_{\boldsymbol{q}}= & 4 J_{1}\left[\cos \left(q_{x} a / 2\right) \cos \left(q_{y} a / 2\right)+\cos \left(q_{y} a / 2\right) \cos \left(q_{z} a / 2\right)\right. \\
& \left.+\cos \left(q_{x} a / 2\right) \cos \left(q_{z} a / 2\right)\right]+2 J_{2}\left[\cos \left(q_{x} a\right)+\cos \left(q_{y} a\right)+\cos \left(q_{z} a\right)\right],
\end{aligned}
$$

where $a$ is the standard lattice parameter. $\mathrm{EuO}$ crystallizes in the $\mathrm{NaCl}$ structure with the lattice constant $a=5.12 \AA$. 


\section{The mode-coupling theory}

In paper [6] the frequency- and wavewector-dependent spin response function was calculated for a Heisenberg ferromagnet using the mode-coupling theory. The results were presented for the critical and paramagnetic regions for EuO. In this theory the following approximations are used:

(a) the projection operator $P$ in the fluctuating force operator

$$
\boldsymbol{f}(t)=\exp [\mathrm{i}(1-P) t L](1-P) \dot{\boldsymbol{S}},
$$

where $\boldsymbol{S}$ is the spin operator, $L$ is the simple Liouville operator and $(\dot{\boldsymbol{S}}=\mathrm{d} \boldsymbol{S} / \mathrm{d} t)$ is neglected,

(b) the dynamic relaxation function of four-spin relaxation functions are approximated by products of two-spin dynamic relaxation functions by means of the dynamic mode-mode decoupling procedure $[6,11]$

$$
\begin{aligned}
& \left(S^{z}(\boldsymbol{k}, t) S^{z}(\boldsymbol{k}-\boldsymbol{q}, t), S^{z}\left(\boldsymbol{k}^{\prime}-\boldsymbol{q}, t\right) S^{z}\left(\boldsymbol{k}^{\prime}\right)\right) \\
& \quad \sim T\left(S^{z}(\boldsymbol{k}, t) S^{z}(-\boldsymbol{k})\right)\left(S^{z}(\boldsymbol{q}-\boldsymbol{k}, t) S^{z}(\boldsymbol{k}-\boldsymbol{q})\right) \delta_{\boldsymbol{k}-\boldsymbol{k}^{\prime}}
\end{aligned}
$$

(c) in order to calculate the static properties of the system, the spherical model will be used because we do not know any simple procedure to calculate, for example, the static susceptibility by means of the mode-coupling theory. The above procedure (b) will be used in the present paper also in the case of the two-pole approximation. The application of the mode-coupling approximation to the study of spin fluctuations in a Heisenberg magnet leads to the following equation for the relaxation function [5-7]:

$$
\begin{aligned}
& \frac{\mathrm{d} F(\boldsymbol{q}, t)}{\mathrm{d} t}=-2 T \sum_{\boldsymbol{k}}(J(\boldsymbol{k})-J(\boldsymbol{q}-\boldsymbol{k}))(J(\boldsymbol{q})-J(\boldsymbol{k})) \chi(\boldsymbol{k}) \\
& \times \int_{0}^{t} \mathrm{~d} t^{\prime}\left(F\left(\boldsymbol{k}, t-t^{\prime}\right) F\left(\boldsymbol{q}-\boldsymbol{k}, t-t^{\prime}\right) F\left(\boldsymbol{q}, t^{\prime}\right),\right.
\end{aligned}
$$

(this formula is true if the Hamiltonian has the form $(1 / 2) \sum_{i j} J_{i, j} \boldsymbol{S}_{i} \boldsymbol{S}_{j}$ ), where the static susceptibility $\chi(T)$ is given by the spherical model $[1,5]$ (see bellow)).

The numerical method employed to solve Eq. (5) has been described in detail by Cuccoli et al. [6]. We recall only that, for the static susceptibility which must be inserted in the theory, we have used the spherical model, because the form is consistent with the dynamical equations. Moreover this susceptibility is in satisfactory agreement with experimental data if the temperature is not too close to $T_{\mathrm{C}}$. The need of having a prescription for the susceptibility is a weakness of the mode-coupling theory, since the prescription is a necessary approximation. We do not know how the uncertainty in the susceptibility influences the usefulness of the theory. A similar dilemma exists in using the three-pole approximation [10]. 
Equation (5) can be written in another form [6]:

$$
\begin{aligned}
& \frac{\mathrm{d} F(\boldsymbol{q}, t)}{\mathrm{d} t}=-T \sum_{\boldsymbol{k}}(J(\boldsymbol{k})-J(\boldsymbol{q}-\boldsymbol{k}))^{2} \chi(\boldsymbol{k}) \chi(\boldsymbol{q}-\boldsymbol{k}) \chi(\boldsymbol{q})^{-1} \\
& \times \int_{0}^{t} \mathrm{~d} t^{\prime}\left(F\left(\boldsymbol{k}, t-t^{\prime}\right) F\left(\boldsymbol{q}-\boldsymbol{k}, t-t^{\prime}\right) F\left(\boldsymbol{q}, t^{\prime}\right) .\right.
\end{aligned}
$$

Finally, we obtain the function $F(\boldsymbol{q}, \omega)$ using a simple fast Fourier (Laplace) transform routine (the numerical method). Taking into account the Laplace transform of (5), we can obtain the known equation for the line-shape function $F(\boldsymbol{q}, z)[4]$ :

$$
F(\boldsymbol{q}, z)=\frac{1}{z+\left(f(\boldsymbol{q}) f^{+}(\boldsymbol{q})\right)_{z} \chi(\boldsymbol{q})^{-1}}, \quad(z=\mathrm{i} \omega),
$$

where the Laplace transformed relaxation function of the random force

$$
(f(\boldsymbol{q}) f(-\boldsymbol{q}))_{z}=\int_{0}^{\infty} \mathrm{e}^{-z t}(f(\boldsymbol{q}, t) f(-\boldsymbol{q})) \mathrm{d} t
$$

(in the mode-mode coupling approximation) is given by the formula (for the Hamiltonian (1))

$$
\begin{aligned}
& \left(f(\boldsymbol{q}) f^{+}(-\boldsymbol{q})\right)_{z} \chi(\boldsymbol{q})^{-1}=\left(\left[S^{z}(\boldsymbol{q}), H\right]\left[S^{z}(\boldsymbol{q}), H\right]^{+}\right) \chi(\boldsymbol{q})^{-1} \\
& \quad=-4 T \chi(\boldsymbol{q})^{-1} \sum_{\boldsymbol{k}}(J(\boldsymbol{k})-J(\boldsymbol{q}-\boldsymbol{k}))^{2} \chi(\boldsymbol{k}) \chi(\boldsymbol{q}-\boldsymbol{k}) \\
& \quad \times \int_{0}^{\infty} \mathrm{e}^{-z t}\left(S^{z}(\boldsymbol{k}, t) S^{z}(\boldsymbol{k})^{+}\right)\left(S^{z}(\boldsymbol{q}-\boldsymbol{k}, t) S^{z}(\boldsymbol{k}-\boldsymbol{q})^{+}\right) \mathrm{d} t .
\end{aligned}
$$

The above equation (9) was obtained by Lindgård [1]. In the case of a two-pole approximation we have $[1,4,12,13]$

$$
\left(f(\boldsymbol{q}) f^{+}(-\boldsymbol{q})\right)_{z} \chi^{-1}(\boldsymbol{q})=\frac{\delta_{1}}{z+2 \beta_{\boldsymbol{q}}},
$$

$\beta_{\boldsymbol{q}}$ is the damping parameter. In the case of the three-pole approximation we have $[4,10]$

$$
\left(f(\boldsymbol{q}) f^{+}(-\boldsymbol{q})\right)_{z} \chi^{-1}(\boldsymbol{q})=\frac{\delta_{1}}{z+\delta_{2} /(z+1 / \tau)},
$$

where $\delta_{1}=\left\langle\omega_{\boldsymbol{q}}^{2}\right\rangle, \delta_{2}=\left(\left\langle\omega_{\boldsymbol{q}}^{4}\right\rangle-\left\langle\omega_{\boldsymbol{q}}^{2}\right\rangle^{2}\right) /\left\langle\omega_{\boldsymbol{q}}^{2}\right\rangle$ are the second and fourth frequency moments of $F(\boldsymbol{q}, \omega)$ and $\tau=\left(\pi \delta_{2} / 2\right)^{-1 / 2}$. The line-shape function $F(\boldsymbol{q}, \omega)$ can be obtained using the following formula:

$$
F(\boldsymbol{q}, \omega)=\frac{1}{\pi} \operatorname{Re} F(\boldsymbol{q}, z),
$$

where 


$$
\int_{\infty}^{-\infty} F \mathrm{~d} \omega=1
$$

(Re denotes real part of $F(\boldsymbol{q}, z)$ ).

\section{The correlation theory within a two-pole approximation}

As it was written in paper [12], the idea of the correlation theory is to calculate both the static and dynamic properties self-consistently, including correlation effects in a mode-mode coupling approximation. The theory has previously been reviewed in detail [13] (all equations are also given in paper [12]). The correlation theory within a two-pole approximation gives the following form for the line-shape function $F(\boldsymbol{q}, \omega)$ (using formulae (10) and (12)):

$$
F(\boldsymbol{q}, \omega)=\frac{1}{\pi} \frac{2 \beta_{\boldsymbol{q}} \delta_{1}}{\left(\omega^{2}-\delta_{1}\right)^{2}+4 \beta_{\boldsymbol{q}}^{2} \omega^{2}}, \quad \delta_{1}=\alpha_{\boldsymbol{q}}^{2}+\beta_{\boldsymbol{q}}^{2}
$$

The damping parameter $\beta_{\boldsymbol{q}}$, in this theory, is determined (see Sec. 6) using a simplest approximation for the Laplace transformed relaxation function of the random force in the form (the paramagnetic state)

$$
\begin{gathered}
(f(\boldsymbol{q}) f(-\boldsymbol{q}))_{z} \chi(\boldsymbol{q})^{-1}=\left(\left[S^{z}(\boldsymbol{q}), H\right],\left[S^{z}(-\boldsymbol{q}), H\right]\right)_{z} \chi(\boldsymbol{q})^{-1}=\frac{\left\langle\omega_{\boldsymbol{q}}^{2}\right\rangle}{z+2 \beta_{\boldsymbol{q}}}, \\
(z=\mathrm{i} \omega)
\end{gathered}
$$

and $\delta_{1}=\left\langle\omega_{\boldsymbol{q}}^{2}\right\rangle$ the second frequency moment of the line-shape function $F(\boldsymbol{q}, \omega)$. Calculating the commutator on the right hand side of (15) for the Hamiltonian (1), we must use the mode-mode approximation for the dynamical relaxation function of four-spin operators [11]. On both sides of (15) static correlation functions appear which are given by the following formula:

$$
\left\langle S^{z}(\boldsymbol{q}) S^{z}(-\boldsymbol{q})\right\rangle=\chi(\boldsymbol{q}) \int_{\infty}^{-\infty} \frac{\omega F(\boldsymbol{q}, \omega) \mathrm{d} \omega}{1-\exp \left(-\omega / k_{\mathrm{B}} T\right)} .
$$

Using the formula for the line-shape function $F(\boldsymbol{q}, \omega)(14)$ we obtain the following formula for the static correlation function by means of the residues (see also [14]):

$$
\begin{gathered}
\left.\left\langle S^{z}(\boldsymbol{q}) S^{z}(-\boldsymbol{q})\right)\right\rangle=\chi(\boldsymbol{q}) k_{\mathrm{B}} T\left[\frac{\alpha_{\boldsymbol{q}}^{2}+\beta_{\boldsymbol{q}}^{2}}{2 k_{\mathrm{B}} T \alpha_{\boldsymbol{q}}} \frac{\sinh \left(\alpha_{\boldsymbol{q}} / k_{\mathrm{B}} T\right)}{\cosh \left(\alpha_{\boldsymbol{q}} / k_{\mathrm{B}} T\right)-\cosh \left(\beta_{\boldsymbol{q}} / k_{\mathrm{B}} T\right)}\right. \\
\left.-\frac{4 \beta_{\boldsymbol{q}} \delta_{1}}{2 \pi k_{\mathrm{B}} T} \sum_{n=1}^{n=\infty} \frac{n}{n^{4}+2 n^{2}\left(\alpha_{\boldsymbol{q}}^{2}-\beta_{\boldsymbol{q}}^{2}\right) /\left(2 \pi k_{\mathrm{B}} T\right)^{2}+\delta_{1}^{2} /\left(2 \pi k_{\mathrm{B}} T\right)^{4}}\right]
\end{gathered}
$$

$\chi(\boldsymbol{q})$ is the static wavevector-dependent susceptibility, $T$ is the temperature, $k_{\mathrm{B}}$ is the Boltzmann constant. From now on we set $k_{\mathrm{B}}$ and also $\hbar$ equal to unity, so energies and frequencies will be in units of K. We notice from (17) that $\left\langle S^{z}(\boldsymbol{q}) S^{z}(-\boldsymbol{q})\right\rangle$ is different from the RPA model which gives $\chi(\boldsymbol{q}) k_{\mathrm{B}} T$. Both models give the 
same results in the limit $T=\infty$. The static equations in the correlation theory for a simple Hamiltonian of the type (1) give the following formula for the static susceptibility [1]:

$$
\chi(\boldsymbol{q})=\frac{\sum_{\boldsymbol{k}}(J(\boldsymbol{k})-J(\boldsymbol{q}-\boldsymbol{k}))\left\langle S^{z}(\boldsymbol{k}) S^{z}(-\boldsymbol{k})\right\rangle}{\sum_{\boldsymbol{k}}(J(\boldsymbol{k})-J(\boldsymbol{q}-\boldsymbol{k}))(J(\boldsymbol{k})-J(\boldsymbol{q}))\left\langle S^{z}(\boldsymbol{k}) S^{z}(-\boldsymbol{k})\right\rangle} .
$$

In the spherical model (RPA method) $\chi(\boldsymbol{q})=\left\langle S^{z}(\boldsymbol{q}) S^{z}(-\boldsymbol{q})\right\rangle / T$, then we can exactly obtain Eq. (33) of paper [5], as it should be. In the case of EuO the above formula can be written in another form:

$$
\chi(\boldsymbol{q})=\frac{1}{J(0) R_{\boldsymbol{q}}-J(\boldsymbol{q})}, \quad R_{\boldsymbol{q}}=\frac{J_{\boldsymbol{q} 2}}{J_{\boldsymbol{q} 1}},
$$

where

$$
\begin{aligned}
J_{\boldsymbol{q} 2} & =4 \alpha_{3}\left[\cos \left(q_{x} a / 2\right) \cos \left(q_{y} a / 2\right)+\cos \left(q_{y} a / 2\right) \cos \left(q_{z} a / 2\right)\right. \\
& \left.+\cos \left(q_{x} a / 2\right) \cos \left(q_{z} a / 2\right)\right]+2 \alpha_{4}\left[\cos \left(q_{x} a\right)+\cos \left(q_{y} a\right)+\cos \left(q_{z} a\right)\right], \\
J_{\boldsymbol{q} 1} & =4 \alpha_{1}\left[\cos \left(q_{x} a / 2\right) \cos \left(q_{y} a / 2\right)+\cos \left(q_{y} a / 2\right) \cos \left(q_{z} a / 2\right)\right. \\
& \left.+\cos \left(q_{x} a / 2\right) \cos \left(q_{z} a / 2\right)\right]+2 \alpha_{2}\left[\cos \left(q_{x} a\right)+\cos \left(q_{y} a\right)+\cos \left(q_{z} a\right)\right] .
\end{aligned}
$$

The parameters $\alpha_{1}, \alpha_{2}, \alpha_{3}, \alpha_{4}$ are expressed in terms of the static correlation functions:

$$
\begin{aligned}
& \alpha_{1}=J_{1} \frac{1}{N} \sum_{\boldsymbol{k}}\left\langle S^{z}(\boldsymbol{k}) S^{z}(-\boldsymbol{k})\right\rangle \cos \left(k_{x} \frac{a}{2}\right) \cos \left(k_{y} \frac{a}{2}\right), \\
& \alpha_{2}=J_{2} \frac{3}{N} \sum_{\boldsymbol{k}}\left\langle S^{z}(\boldsymbol{k}) S^{z}(-\boldsymbol{k})\right\rangle \cos \left(k_{x} \frac{a}{2}\right), \\
& \alpha_{3}=J_{1} \frac{1}{N} \sum_{\boldsymbol{k}}\left\langle S^{z}(\boldsymbol{k}) S^{z}(-\boldsymbol{k})\right\rangle \cos \left(k_{x} \frac{a}{2}\right) \cos \left(k_{y} \frac{a}{2}\right) \frac{J(\boldsymbol{k})}{J(0)}, \\
& \alpha_{4}=J_{2} \frac{3}{N} \sum_{\boldsymbol{k}}\left\langle S^{z}(\boldsymbol{k}) S^{z}(-\boldsymbol{k})\right\rangle \cos \left(k_{x} a\right) \frac{J(\boldsymbol{k})}{J(0)} .
\end{aligned}
$$

The second frequency moment $\delta_{1}$ of the line-shape function $F(\boldsymbol{q}, \omega)$ can be expressed in terms of the static correlation functions:

$$
\chi(\boldsymbol{q}) \delta_{1}=2\left(J_{01}-J_{\boldsymbol{q} 1}\right) .
$$

Because, the static correlation function (17) is expressed by the parameters $\alpha_{i}(i=1, \ldots, 4)$ through the second frequency moment and $\chi(\boldsymbol{q})$, therefore these parameters can be calculated in a self-consistent way, solving a set of equa- 
tions (22), by means of the Newton method (for a fixed value of $\beta_{\boldsymbol{q}}$ ). From (19) $\chi(0)$ is given by the following formula:

$$
\chi(0)=\frac{1}{J(0)\left(\frac{6 \alpha_{3}+\alpha_{4}}{6 \alpha_{1}+\alpha_{2}}-1\right)} .
$$

The critical temperature $T_{\mathrm{C}}$ is a temperature at which $\chi(0)$ diverges

$$
\frac{6 \alpha_{3}+\alpha_{4}}{6 \alpha_{1}+\alpha_{2}}=1
$$

It should be noted that the conditions (25) and Eq. (24) are true also in the case of the RPA method, when the parameters $\alpha_{i}=\alpha_{i}(T),(i=1, \ldots, 4)$ are calculated by means of this method.

For $T>T_{\mathrm{C}}$ the line-shape function $F(\boldsymbol{q}, \omega)$ (14) has two resonances, namely at $\omega_{\boldsymbol{q}}= \pm \alpha_{\boldsymbol{q}}+\mathrm{i} \beta_{\boldsymbol{q}}$. For $\alpha_{\boldsymbol{q}}>\beta_{\boldsymbol{q}}$ we have two distinct peaks. These are called the sloppy spin waves in the paramagnetic phase, as observed in EuO. The "sloppy spin wave frequency" is at $\omega_{\text {peak }}= \pm \sqrt{\alpha_{\boldsymbol{q}}^{2}-\beta_{\boldsymbol{q}}^{2}}$. The spin waves are overdamped for $\alpha_{\boldsymbol{q}}<\beta_{\boldsymbol{q}}$ and $\omega_{\text {peak }}=0$. Because in paper [15] the formulae for the lineshape function $F(\boldsymbol{q}, \omega)$ were compared in both cases, the two-pole approximation and double Lorentzian approximations (for small values of $\boldsymbol{q}$ and $T=66 \mathrm{~K}$, here $T_{\mathrm{C}}=69.4 \mathrm{~K}$ ), by fitting to the experimental data of spin waves in EuO, therefore below, the formula for $F(\boldsymbol{q}, \omega)$ will be given in the case of double Lorentzian form (for $T<T_{\mathrm{C}}$ )

$$
F_{\mathrm{LOR}}(\boldsymbol{q}, \omega)=\frac{2}{\pi}\left(\frac{\Gamma_{\boldsymbol{q}}}{\Gamma_{\boldsymbol{q}}^{2}+\left(\omega-\omega_{\mathrm{peak}}\right)^{2}}+\frac{\Gamma_{\boldsymbol{q}}}{\Gamma_{\boldsymbol{q}}^{2}+\left(\omega+\omega_{\mathrm{peak}}\right)^{2}}\right) .
$$

Here the damping parameter $\beta_{\boldsymbol{q}}$ and $\Gamma_{\boldsymbol{q}}$ are related to lifetime of excitations. The diffusive scattering case is often approximated by a simple Lorentzian

$$
F_{\mathrm{LOR}}(\boldsymbol{q}, \omega)=\frac{1}{\pi}\left(\frac{\Gamma_{\boldsymbol{q}}}{\Gamma_{\boldsymbol{q}}^{2}+\omega^{2}}\right) .
$$

The wavelength-dependent susceptibility $\chi(\boldsymbol{q})$ is usually expanded to the second order in $\boldsymbol{q}$ as

$$
\chi(\boldsymbol{q}) \sim \frac{1}{\kappa_{1}^{2}+q^{2}},
$$

this defines the correlation length $\xi^{-1}=\kappa_{1}$. 


\section{The cut-off two-pole approximation}

In paper [1] there was used the cut-off two-pole approximation, in which the line-shape $F(\boldsymbol{q}, \omega)$ is cut-off at frequency $\omega_{\mathrm{c}}$ determined by the fourth frequency moment $\left\langle\omega_{\boldsymbol{q}}^{4}\right\rangle$ (see appendix A). This function has the harmonic oscillator form with poles $\omega \pm \alpha_{\boldsymbol{q}}+\mathrm{i} \beta_{\boldsymbol{q}}$ :

$$
F(\boldsymbol{q}, \omega)= \begin{cases}\frac{k}{\pi}\left[\left(\omega^{2}-a_{\boldsymbol{q}}^{2}\right)^{2}+4 \beta_{\left.\boldsymbol{q}^{2} \omega^{2}\right]^{-1},}\right. & -\omega_{\mathrm{c}}<\omega<\omega_{\mathrm{c}} \\ 0 & \text { otherwise, }\end{cases}
$$

where $a_{\boldsymbol{q}}^{2}=\alpha_{\boldsymbol{q}}^{2}+\beta_{\boldsymbol{q}}^{2}$.

$F(\boldsymbol{q}, \omega)$ must be normalized such that $\int_{-\omega_{\mathrm{c}}}^{\omega_{\mathrm{c}}} F(\boldsymbol{q}, \omega) \mathrm{d} \omega=1$. And the static correlation function $\left\langle S^{z}(\boldsymbol{q}) S^{z}(-\boldsymbol{q})\right\rangle$ is determined by the following formula:

$$
\left\langle S^{z}(\boldsymbol{q}) S^{z}(-\boldsymbol{q})\right\rangle=\chi(\boldsymbol{q}) \frac{k}{\pi} \int_{-\omega_{\mathrm{c}}}^{\omega_{\mathrm{c}}} \frac{\omega F(\boldsymbol{q}, \omega) \mathrm{d} \omega}{1-\exp (-\omega / T)} .
$$

The frequency moments are given by the formula $\left\langle\omega_{\boldsymbol{q}}^{n}\right\rangle=\int_{-\omega_{c}}^{\omega_{c}} \omega^{n} F(\boldsymbol{q}, \omega) \mathrm{d} \omega$, $n=2,4$. In paper [1] the equations are given which describe the cut-off frequency $\omega_{\mathrm{c}}$, the frequency moments $\left\langle\omega_{\boldsymbol{q}}^{2}\right\rangle,\left\langle\omega_{\boldsymbol{q}}^{4}\right\rangle$, and also the parameters $\alpha_{\boldsymbol{q}}$ and $k$. These equations are solved iteratively for fixed $\left\langle\omega_{\boldsymbol{q}}^{2}\right\rangle,\left\langle\omega_{\boldsymbol{q}}^{4}\right\rangle$, and $\beta_{\boldsymbol{q}}$ (see appendix A).

\section{The damping parameter}

The wavevector and temperature dependence of the damping parameter $\beta_{\boldsymbol{q}}$ can be obtained using Eqs. (15) and (9) for Hamiltonian (1) as well as using the following approximations [1]:

(a) calculating the commutators on the right hand side of Eq. (15) we obtain the dynamic relaxation function of the four operators which we approximate by products of the dynamic relaxation function of two-spin operators by means of the mode-mode decoupling procedure [11];

(b) under the sum over $\boldsymbol{k}$ and the integral over $t$, the precise frequency dependence of the Laplace transform of the relaxation function of two-spin operators $\left(S^{z}(\boldsymbol{k}, t) S^{z}(-\boldsymbol{k})\right)$ on the right hand side of the formula (9) is not important. It is therefore justified to approximate (9) further by a simple Lorentzian line-shape (which can easily be Laplace transformed)

$$
\left(\left(S^{z}(\boldsymbol{k}) S^{z}(-\boldsymbol{k})\right)_{z}=\frac{\chi(\boldsymbol{k})}{z+\Gamma_{\boldsymbol{k}}}, \quad\left(S^{z}(\boldsymbol{k}, t) S^{z}(-\boldsymbol{k})\right) \sim \chi(\boldsymbol{k}) \mathrm{e}^{-\Gamma_{\boldsymbol{k}} t},\right.
$$

with the same area and halfwidth as the cut-off two-pole line-shape (14). The halfwidth frequency is then given by:

$$
\Gamma_{\boldsymbol{k}}^{2}=\alpha_{\boldsymbol{k}}^{2}-\beta_{\boldsymbol{k}}^{2}+\left[2\left(\alpha_{\boldsymbol{k}}^{4}+\beta_{\boldsymbol{k}}^{4}\right)\right]^{1 / 2}, \quad \alpha_{\boldsymbol{k}}^{2}=\left\langle\omega_{\boldsymbol{k}}^{2}\right\rangle-\beta_{\boldsymbol{k}}^{2} .
$$


Using (15) and (10) and the approximation (31) one obtains the following equation for the self-consistent determination of $\beta_{\boldsymbol{q}}$ :

$$
\frac{\chi(\boldsymbol{q})\left\langle\omega_{\boldsymbol{q}}^{2}\right\rangle}{\Gamma_{\boldsymbol{q}}+2 \beta_{\boldsymbol{q}}}=\frac{4 k_{\mathrm{B}} T}{N}\left[\sum_{\boldsymbol{k}}(J(\boldsymbol{k})-J(\boldsymbol{k}-\boldsymbol{q}))^{2} \frac{\chi(\boldsymbol{q}-\boldsymbol{k}) \chi(\boldsymbol{k})}{\Gamma_{\boldsymbol{q}}+\Gamma_{\boldsymbol{k}}+\Gamma_{\boldsymbol{q}-\boldsymbol{k}}}\right] .
$$

Finally, as was written in [1], the wavevector dependence for the damping parameter $\beta_{\boldsymbol{q}}$ was found for the fcc lattice to be well represented by a Fourier expansion

$$
\beta_{\boldsymbol{q}}^{2}=\sum_{n=0}^{3} b_{n}\left(1-\frac{J(0)}{J(\boldsymbol{q})}\right)^{n},
$$

where $b_{n}$ are temperature-dependent constants which can be found in terms of the static properties by considering four wavevectors from the Brillouin zone in Eq. (33) (near $T_{\mathrm{C}}$ it may be better to expand $\beta_{\boldsymbol{q}}^{-1}$ in $(34)[1,13]$ ).

\section{The three-pole approximation within RPA method}

From the theoretical point of view, it is well known that in the continued-fraction theory [4] the three-pole approximation is the obvious mathematical next step beyond the two-pole approximation in which line-shape function $F(\boldsymbol{q}, \omega)$ is given by

$$
F(\boldsymbol{q}, \omega)=\frac{1}{\pi} \frac{\tau \delta_{1} \delta_{2}}{\left[\omega \tau\left(\omega^{2}-\delta_{1}-\delta_{2}\right)\right]^{2}+\left(\omega^{2}-\delta_{1}\right)^{2}},
$$

where

$$
\delta_{1}=\left\langle\omega_{\boldsymbol{q}}^{2}\right\rangle, \quad \delta_{1} \delta_{2}=\left\langle\omega_{\boldsymbol{q}}^{4}\right\rangle-\left\langle\omega_{\boldsymbol{q}}^{2}\right\rangle^{2},
$$

$\left\langle\omega_{\boldsymbol{q}}^{2}\right\rangle,\left\langle\omega_{\boldsymbol{q}}^{4}\right\rangle$ are the second and fourth frequency moments of $F(\boldsymbol{q}, \omega)$,

$$
\tau=\left(\pi \delta_{2}^{2} / 2\right)^{-1 / 2}
$$

In paper [10] all quantities, which describe static properties of EuO, were evaluated by the spherical approximation (RPA method). $\left\langle\omega_{\boldsymbol{q}}^{2}\right\rangle$ and $\left\langle\omega_{\boldsymbol{q}}^{4}\right\rangle$ can be expressed in terms of the static correlation functions (see also the formulae (18) and (19))

$$
T \chi(\boldsymbol{q})=\left\langle S^{z}(\boldsymbol{q}) S^{z}(-\boldsymbol{q})\right\rangle=\frac{T}{J(0)-J(\boldsymbol{q})+\chi(\boldsymbol{q}=0)^{-1}} .
$$

The magnetic susceptibility $\chi(\boldsymbol{q}=\mathbf{0})=\chi(0)$ is determined by the sum rule

$$
\frac{1}{N} \sum_{\boldsymbol{q}}\left\langle S^{z}(\boldsymbol{q}) S^{z}(-\boldsymbol{q})\right\rangle=\frac{1}{3} S(S+1) \text {. }
$$


At $T_{\mathrm{C}}$ one has $\chi(0)=0$ so the transition occurs when

$$
\frac{1}{N} \sum_{\boldsymbol{k}} \frac{T_{\mathrm{C}}}{J(0)-J(\boldsymbol{k})}=\frac{1}{3} S(S+1) .
$$

The shape function $F(\boldsymbol{q}, \omega)$ (35) has either one peak $\omega=0$ if $\delta_{2}>2 \delta_{1}$ or three peaks, at $\omega=0$, and $\omega= \pm \omega_{\text {sw }}$ if $\delta_{2}<2 \delta_{1}$. We shall call maximum at $\omega=\omega_{\mathrm{sw}}$ the "spin wave" peak. Finally, to understand the structure of $F(\boldsymbol{q}, \omega)$ we better rewrite it in a more transparent form

$$
F(\boldsymbol{q}, \omega)=\frac{\frac{1}{\pi} \gamma \omega_{0}^{2}}{\left(\omega^{2}-\omega_{0}^{2}\right)+\gamma^{2} \omega^{2}\left[\left(\omega^{2}-\omega_{0}^{2}-\frac{\pi}{2} \gamma^{2}\right) / \frac{\pi}{2} \gamma^{2}\right]^{2}},
$$

with $\gamma=\left(2 \delta_{2} / \pi\right)^{1 / 2}$ and $\omega_{0}^{2}=\delta_{1}$. Equation (40) resembles a damped harmonic oscillator (14). They have an identical form.

\section{Results and comparison with experiment}

First of all we describe the available experimental data. The experimental values of the reduced temperature dependence of the susceptibility $\chi(T) / \chi(0)$ (where $\chi(0)=S(S+1) /(3 T)$ ) of $\mathrm{EuO}$ is given in paper [3]. The observed value of $T_{\mathrm{C}}$ was $69.25 \pm 0.05 \mathrm{~K}$. In [2] the Curie temperature was determined by measuring the temperature dependence of the critical scattering and the value $T_{\mathrm{C}}=69.25 \pm 0.05 \mathrm{~K}$ was obtained. Using a simple Lorentzian function $(27)$ the linewidth $\Gamma_{\boldsymbol{q}}$ was also determined in the form $\Gamma_{\boldsymbol{q}}=A q^{2.5}$, where $A=8.3 \mathrm{meV} \AA^{2.5}$ for $q<0.3 \AA^{-1}$. In the small- $q$ region it is more appropriate to compare the linewidth measurements with the dynamical scaling approach performed by Riedel and Hubbard who predicted $A=6.4 \mathrm{meV}^{5 / 2}$ [17] and $7.0 \mathrm{meV} \AA^{5 / 2}$ [5], respectively. These values are in good agreement with the experimental value. In [3] from inelastic neutron scattering above $T_{\mathrm{C}}$ there were obtained: $T_{\mathrm{C}}=69.25 \pm 0.05 \mathrm{~K}$, the relation $\chi(\boldsymbol{q}) / \chi(0)=C\left[\left(T-T_{\mathrm{C}}\right) / T_{\mathrm{C}}\right]^{-\gamma}$ (where the critical parameter $\gamma=1.387)$, and also the formula for the inverse spin correlation range $\kappa_{1}$ in the form $a_{n m} \kappa_{1}=F^{+}\left[\left(T-T_{\mathrm{C}}\right) / T_{\mathrm{C}}\right]^{\nu}$, where $a_{n m}=3.64 \AA$ is defined to be the nearest-neighbour distance, the constants $\nu$ and $F^{+}$have values: $\nu=0.681 \pm 0.17$ and $F^{+}=2.32 \pm 0.13$. Mook [8] presents spectra for the relaxation function $\left(S^{z}(\boldsymbol{q}) S^{z}(-\boldsymbol{q})\right)_{\omega}=\chi(\boldsymbol{q}) F(\boldsymbol{q}, \omega)$ at $T / T_{\mathrm{C}}=1.0,1.27$, and 2.0. At each of these temperatures "spin wave" peaks are seen in the $[1,1,1]$ direction for $q / q_{\max }=1.0$ but not for $q / q_{\max }=0.5$, where $q_{\max }$ is the zone-boundary wavevector in the direction considered. As was noted in [2] the experimental value of $q_{\max }$ is $q_{\max }=1.06 \AA^{-1}$ and the theoretical value is $q_{\max }=1.0 \AA^{-1}$. In paper [1] the calculated temperature dependence of the magnetic susceptibility $\chi(T) / \chi(0)$ was presented using the formula of type (24). The theoretical values of $\chi(T) / \chi(0)$ were compared to the experimental results. The agreement between theory and experiment is quite satisfactory. In [16] the experimental values of $\delta_{1}$ and $\delta_{2}$ from formula (35) were 
obtained by fitting $F(\boldsymbol{q}, \omega)$ to experimental data. The theoretical values for these parameters were calculated by means of the spherical model (38)-(40). It should be noted that these parameters are almost identity at the zone-boundary for $T=T_{\mathrm{C}}$ and $T=1.68 T_{\mathrm{C}}$. It should be also noted that in paper [16] the paramagnetic experimental peaks were obtained in the spectrum for $0.7 \AA^{-1}$ and $1.0 \AA^{-1}$ at $T=T_{\mathrm{C}}$ and at $T=1.68 T_{\mathrm{C}}(116 \mathrm{~K})$. These peaks were very well described by means of the three-pole approximation. In this paper the calculated values of the line-shape function $F(\boldsymbol{q}, \omega)$ were plotted for $[1,1,1],[1,1,0]$, and $[1,0,0]$ directions. It should be noted that the scattering is very isotropic, in fact, the peak positions do not deviate by more than half the resolution width $(0.4 \mathrm{meV})$ from each other. In [15] the data from the polarized neutron experiments were fitted to both a two-pole approximation (14) and double Lorentzian (26) forms for $F(\boldsymbol{q}, \omega), T<T_{\mathrm{C}}$ (here $T=66 \mathrm{~K}$ and $\left.T_{\mathrm{C}}=69.4 \mathrm{~K}\right)$. The two-pole approximation (14) gave a significantly better fit.

\section{TABLE I}

Calculated MFA, RPA, CT, and measured transition temperatures in K. MFA mean field approximation, RPA - random phase approximation, CT - correlation theory, exp - experiment $[2,3]$.

\begin{tabular}{c|c|c|c}
\hline \hline$T_{\mathrm{C}}(\mathrm{MFA})$ & $T_{\mathrm{C}}(\mathrm{RPA})$ & $T_{\mathrm{C}}(\mathrm{CT})$ & $T_{\mathrm{C}}(\exp )$ \\
\hline 86.6 & 66.5 & 66.6 & 69.2
\end{tabular}

TABLE II

Calculated values of the energy of the "spin wave" peak $\omega_{\mathrm{sw}}$ (in $\operatorname{meV})$ for $q=q_{\max }$ in $[1,1,1]$ direction at different values of $T / T_{\mathrm{C}}$ ( $q_{\max }$ is the zone-boundary wavevector in this direction). The experimental results are from Mook [8].

\begin{tabular}{c|c|c|c}
\hline \hline$T / T_{\mathrm{C}}$ & 1.0 & 1.27 & 2.0 \\
\hline$\omega_{\text {sw }}\left(q_{\max }\right)$, simple two-pole approximation & 18.0 & 12.15 & 7.9 \\
\hline$\omega_{\text {sw }}\left(q_{\max }\right)$, cut-off two-pole approximation & 23.1 & 17.1 & 13.0 \\
\hline$\omega_{\text {sw }}\left(q_{\max }\right)$, three-pole approximation & 32.5 & 25.0 & 20.0 \\
\hline experiment & 32 & 25 & 23
\end{tabular}

Now, let us describe our results. In Table I the theoretical and experimental values of the critical values $T_{\mathrm{C}}$ are presented for $\mathrm{EuO}$ obtained using the formula (25), for RPA, and correlation theory, and in the case of mean field approximation (MFA), the simple formula $T_{\mathrm{C}}=(1 / 3) S(S+1) J(0)[10]$. In Table II the calculated values of the energy of the "spin wave" peak $\omega_{\text {sw }}$ are presented for 
TABLE III

Calculated values of the wavevector $q^{*} / q_{\max }$ beyond which a "spin wave" peak in the shape function $F(\boldsymbol{q}, \omega)$ is observed as a function of $\omega$ and $\boldsymbol{q}$ in $[1,1,1]$ direction at different of $T / T_{\mathrm{C}}$. The experiment results are from Mook [8].

\begin{tabular}{c|c|c|c}
\hline \hline$T / T_{\mathrm{C}}$ & 1.0 & 1.27 & 2.0 \\
\hline$q^{*}$, simple pole approximation & 0.60 & 0.64 & 0.73 \\
\hline$q^{*}$, cut-off two-pole approximation & 0.52 & 0.54 & 0.59 \\
\hline$q^{*}$, three-pole approximation & 0.68 & 0.76 & 0.82 \\
\hline$q^{*}$, experiment & between & between & between \\
& $0.5-1.0$ & $0.5-1.0$ & $0.5-1.0$
\end{tabular}

$q=q_{\max }$ in $[1,1,1]$ direction at different values of $T / T_{\mathrm{C}}$. In Table III there are presented the calculated values of the wavevector $q^{*}$ above which a "spin wave" peak is observed in the shape function $F(\boldsymbol{q}, \omega)$ as a function of $\omega$ and $\boldsymbol{q}$ at different values of $T / T_{\mathrm{C}}$. Looking at Tables II and III one can say that there is a better overall agreement between the calculated and experimental values in the case of the three-pole approximation than the two-pole approximation. From the theoretical point of view, it is well known that in a continued fraction theory [4] a three-pole approximation is the obvious mathematical next step beyond the twopole approximation. As it noted by Lindgård [13], the introduction of the cut-off two-pole approximation does not influence any of the qualitative results, however, the quantitative values for $\alpha_{\boldsymbol{q}}$ and $\beta_{\boldsymbol{q}}$ (in Eqs. (14), (17)) will be somewhat changed, and so also, the predicted values of the positions of the peaks that appear in the spectrum. Our calculations show the quantitative difference between these approximations for $\mathrm{EuO}$ in predicting the shape of the spectrum. However, in the case of three-pole approximation the frequency moments were calculated here in the approximate way, by means of the RPA method (the spherical approximation) and in the case of a two-pole approximation the second frequency moment is calculated in a self-consistent way. All considered theories predict a "spin wave" peak in the spectrum only for $q>0.5 q_{\max }$. This is in agreement with experiment. Finally, it should be noted also that, in the present paper, the correlation theory was presented in the simple and analytic form (see the formulae (14), (17), (19), (23)-(25)). The simple and analytic formulae (14), (17), (19), (23)-(25) can be derived for all magnetic materials, where the magnetic properties are described by the Hamiltonian of type (1). Finally, it should be emphasised that:

(a) in the case of both the mode-coupling theory and three-pole approximation for dynamical relaxation function we do not know a simple procedure to calculate the static properties of the system by means of the given approximation, therefore we must use the spherical method (RPA method); 
(b) in the case of a two-pole approximation we do not know the wavevector and temperature dependence of the damping parameter $\beta_{\boldsymbol{q}}$, therefore we must use the approximate relation $\beta_{\boldsymbol{q}}$ of type (34);

(c) the presented form of the correlation theory within a two-pole approximation has a simple form from the numerical point of view. The obtained equations in this theory can be solved by means of the simple Newton method.

\section{Acknowledgments}

We are grateful to Prof. Per-Anker Lindgård and A. Kowalska for helpful discussion of the correlation theory within a two-pole approximation.

\section{Appendix A}

In paper [1] (appendix B) there are the editorial errors in the equations which determine the temperature and wavevector dependence of the following formulae: the second frequency moment $\left\langle\omega_{\boldsymbol{q}}^{2}\right\rangle$, the cut-off frequency $\omega_{\mathrm{c}}$, and the constant $k$ (in the case of the line-shape function $F(\boldsymbol{q}, \omega)$ of the type (29)), therefore, below, we give the correct formulae as follows:

$$
\omega_{\mathrm{c}}=\frac{\pi}{2 k}\left[\left\langle\omega_{\boldsymbol{q}}^{4}\right\rangle-2\left(\alpha_{\boldsymbol{q}}^{2}-\beta_{\boldsymbol{q}}^{2}\right)\left\langle\omega_{\boldsymbol{q}}^{2}\right\rangle+a_{\boldsymbol{q}}^{4}\right]
$$

where

$$
\begin{aligned}
k= & 4 \pi \beta_{\boldsymbol{q}} a_{\boldsymbol{q}}^{2}\left\{\frac{\beta_{\boldsymbol{q}}}{\alpha_{\boldsymbol{q}}} \ln \frac{\omega_{\mathrm{c}}^{2}+2 \alpha_{\boldsymbol{q}} \omega_{\mathrm{c}}+a_{\boldsymbol{q}}^{2}}{\omega_{\mathrm{c}}^{2}-2 \alpha_{\boldsymbol{q}} \omega_{\mathrm{c}}+a_{\boldsymbol{q}}^{2}}\right. \\
& \left.+2\left[\arctan \left(\frac{\omega_{\mathrm{c}}-\alpha_{\boldsymbol{q}}}{\beta_{\boldsymbol{q}}}\right)+\arctan \left(\frac{\omega_{\mathrm{c}}+\alpha_{\boldsymbol{q}}}{\beta_{\boldsymbol{q}}}\right)\right]^{-1}\right\}, \quad \alpha_{\boldsymbol{q}}^{2}>0 \\
k= & 2 \pi \beta_{\boldsymbol{q}} a_{\boldsymbol{q}}^{2} \alpha_{11}\left[\left(\beta_{\boldsymbol{q}}+\alpha_{11}\right) \arctan \frac{\omega_{\mathrm{c}}}{\beta_{\boldsymbol{q}}-\alpha_{11}}\right. \\
& \left.-\left(\beta_{\boldsymbol{q}}-\alpha_{11}\right) \arctan \frac{\omega_{\mathrm{c}}}{\alpha_{11}+\beta_{1}}\right]^{-1}, \quad a_{\boldsymbol{q}}^{2}<0,
\end{aligned}
$$

and

$$
\begin{aligned}
& \left\langle\omega_{\boldsymbol{q}}^{2}\right\rangle=a_{\boldsymbol{q}}^{2}\left[\frac{k}{\pi \beta_{\boldsymbol{q}} a_{\boldsymbol{q}}^{2}}\left(\arctan \frac{\omega_{\mathrm{c}}-\alpha_{\boldsymbol{q}}}{\beta_{\boldsymbol{q}}}+\arctan \frac{\omega_{\mathrm{c}}+\alpha_{\boldsymbol{q}}}{\beta_{\boldsymbol{q}}}\right)-1\right], \alpha_{\boldsymbol{q}}^{2}>0, \\
& \left\langle\omega_{\boldsymbol{q}}^{2}\right\rangle=\frac{k}{\pi \beta_{\boldsymbol{q}}}\left[\arctan \frac{\omega_{\mathrm{c}}}{\alpha_{11}+\beta_{\boldsymbol{q}}}+\arctan \frac{\omega_{\mathrm{c}}}{\alpha_{11}-\beta_{\boldsymbol{q}}}\right]+\alpha_{11}^{2}-\beta_{\boldsymbol{q}}^{2}, \alpha_{\boldsymbol{q}}^{2}<0,
\end{aligned}
$$

where $\alpha_{\boldsymbol{q}}=\mathrm{i} \alpha_{11}$ when $\alpha_{\boldsymbol{q}}^{2}<0$ and $a_{\boldsymbol{q}}^{2}=\alpha_{\boldsymbol{q}}^{2}+\beta_{\boldsymbol{q}}^{2}$. If $\omega_{\mathrm{c}} \rightarrow \infty$ then $k=2 \beta_{\boldsymbol{q}} a_{\boldsymbol{q}}^{2}$ and $\left\langle\omega_{\boldsymbol{q}}^{2}\right\rangle=a_{\boldsymbol{q}}^{2}$ (it is exactly a two-pole approximation, see (14)), as it should be. 


\section{References}

[1] P.A. Lindgård, Phys. Rev. B 27, 2980 (1983).

[2] P. Böni, M.E. Chen, G. Shirane, Phys. Rev. B 35, 8849 (1987).

[3] J. Als-Nielsen, O.W. Dietrich, L. Passel, Phys. Rev. B 14, 4908 (1976).

[4] H. Mori, Prog. Theor. Phys. 34, 399 (1965).

[5] J. Hubbard, J. Phys. C 4, 51 (1971).

[6] A. Cuccoli, V. Tognetti, S.W. Lovesey, Phys. Rev. B 39, 2619 (1989).

[7] A. Cuccoli, V. Tognetti, S.W. Lovesey, J. Phys., Condens. Matter 2, 3339 (1990).

[8] H.A. Mook, Phys. Rev. Lett. 46, 508 (1981).

[9] R. Chaudury, B.S. Shastry, Phys. Rev. B 37, 5216 (1988).

[10] A.P. Young, B.S. Shastry, J. Phys. C 15, 4547 (1980).

[11] K. Kawasaki, in: Phase Transition and Critical Phenomena, Eds. C. Domb, M.S. Green, Vol. 5a, Academic Press, London 1976, p. 165.

[12] P.A. Lindgård, O.G. Mouritsen, Phys. Rev. B 41, 688 (1990).

[13] P.A. Lindgård, Spin Waves and Magnetic Excitions, Eds. A.J. Borovik-Romanov, S.K. Sinha, Vol. 22.1, North-Holland, New York 1988, Ch. 5; P.A. Lindgård, Phys. Rev. B 30, 2779 (1984).

[14] P.A. Lindgård, D. Yang, J. Magn. Magn. Mater. 15, 1037 (1980).

[15] R.G. Lloyd, S.M. Hayden, P.W. Mitchell, H.A. Mook, Physica B 156-157, 235 (1989).

[16] P. Böni, G. Shirane, Phys. Rev. B 33, 3012 (1986).

[17] E.R. Riedel, J. Appl. Phys. 40, 1197 (1971). 